Ferraro. D. P. Effects of $\Delta^{s}$-tetrahydrocannabinol on simple and complex learned behavior in animals. In M. F. Lewis (Ed.). Current research in marijuana. New York: Academic Press. 1972. Pp. 46-96.

Glick. S. D.: \& Milloy, S. Aversive effects of $\Delta^{9}$-THC. Psychonomic Science. 1972, 29.6.

Grisham. M. G.. \& Ferraro. D. P. Biphasic effects of $د^{9}-$ THC on variable interval schedule performance in rats. Psychopharmacologia. 1972. 27. 193.

Lyons. J. E.. Klipec. W. D.. \& Eirick. R. The effect of chlorpromazine on the peak shift in male albino rat. Physiological Psychology. 1973. 1. 165-168.

Lyons. J. E.. Klipec. W. D.. \& Steinsultz. G. The effect of chlorpromazine on discrimination performance and the peak shift. Physiological Psychology. 1973. 1. 121-124.
Terrace, H. S. Br-products of discrimination learning. In G. H. Bower (Ed.). The psichology of learning and motivation vew York: Academic Press. 1972. Pp. 195-265.

\section{NOTE}

1. Synthetic $د^{9}$-THC was made available in a dehydrated alcohol solution by Dr. Monique Braude of the NIMH. All doses were taken from NIMH Batch SSC-69060 $\left(93^{\digamma_{c}} \mathcal{J}^{9}-\right.$ THC. $3.2 \tau_{c}$ $J^{\circ}$-THC and $3.1 c_{c}$ cannabinol). The drug vehicle consisted of $0.975 \mathrm{cc}$ isotonic saline, $0.020 \mathrm{cc}$ dehydrated ethyl alcohol. and 0.005 cc Tween 80 .

(Received for publication June 18. 1973.)

\title{
Food intake as a function of age and food deprivation*
}

\author{
LEONARD F. JAKUBCZAK \\ Gerontological Psichology Laboratory. I'A Hospital \\ Jefferson Barracks Division. St. Louis. .Mo. 63125
}

The purpose of this experiment was to determine whether or not there were age differences in the effects of increasing durations of food deprivation on food intake in rats. The experiment was carried out according to a 3 by 3 by 3 by 7 mixed design consisting of three age groups of 12 female Sprague-Dawley rats each (4, 11 , and 24 months), subdivided into three sequence-effects control groups ( $\mathrm{ABC}, \mathrm{BCA}, \mathrm{CAB})$, with each rat exposed to each food-deprivation duration (24, 48 , and $96 \mathrm{~h}$ ), and refeeding measured for 7 days. The dependent variables were: latency to eat, amount of

*This investigation was supported by 821 funds of the Veterans Administration. The author acknowledges the competent technical assistance of J. Hoefel. David Ritchie, and Vern II Intosh. food per day consumed during refeeding, and body weight. Prior to food deprivation, the 4-month-old rats ate less than the 11-month-old rats or the 24-month-old rats, but these differences were proportional to age differences in body weight. The effects of duration of deprivation, order, and days of refeeding were proportional across age, suggesting equal responsivity to the effects of these factors on food intake across a large segment of the life span of the female rat.

The purpose of this experiment was to determine whether or not there were age differences in the effects of increasing durations of food deprivation on the food intake of rats. On the basis of indirect evidence. such as crossings in the Columbia obstruction apparatus. Jerome (1959) concluded that the degree of hunger consequent upon a given duration of food deprivation was inversely related to age. In view of the lack of direct evidence. it 


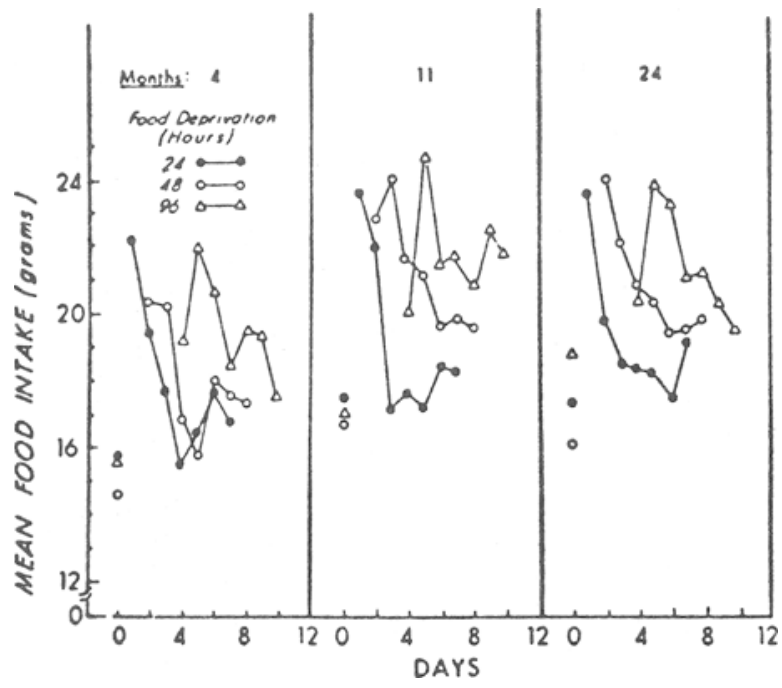

Fig. 1. Food intake as a function of hours of food deprivation, age, and days, averaged over order.

was desirable to investigate the effects of acute food deprivation on rats that represent a large age range, using measures that are more direct indices of the hunger drive.

\section{METHOD}

\section{Procedure}

The experiment was carried out according to a 3 by 3 by 3 by 7 factoral design (Case I. Winer. 1971) consisting of three age groups of 12 female Sprague-Dawley rats each $(4,11$, and 24 months), subdivided into three sequence-effects control groups (A-24, 48, $96 \mathrm{~h} ; \mathrm{B}-48,96,24 \mathrm{~h}$; and C-96, 24, $48 \mathrm{~h}$ ), with each rat exposed to each food-deprivation duration $(24,48$, and $96 \mathrm{~h}$ ) and refeeding measured for 7 days. The rats had been obtained from the Animal Production Section, National Institutes of Health. The sequence factor was included in the design of the experiment in order to assess potential sequence effects of the duration of deprivation. The dependent variables consisted of body weight in grams, the latency to eat in minutes. and the number of grams of food consumed per day during 7 days of refeeding.

Twenty days before the beginning of the food deprivation, the rats were placed in individual $7 \times 10 \times 7$ in. cages (Aloe V2177A), containing a water bottle and spill-resistant foodcup (Wahmann LC306A) in which powdered Purina Chow was dispensed. Prior to this, the rats had been housed in group cages (Aloe V2177B), two to five to a cage. and fed Purina Chow pellets. The cages were located in an air-conditioned room regulated at approximately $75^{\circ} \mathrm{F}$, with at least $8 \mathrm{~h}$ of light a day. Both the animal and the foodcup were weighed daily. except as dictated by the experimental requirements. Water was available to the rat ad lib throughout the experiment.

On the scheduled day and hour, the foodcups of the appropriate rats were removed. Body weights continued to be determined daily. Exactly 24. 48, or $96 \mathrm{~h}$ later the rat was weighed and placed in a $19 \times 11 \times 6$ in. cage (Aloe VMP-43 with VMP-42H lid) in order to determine the latency to eat. The rat was placed at the end of the cage under the V-shaped feeder (empty). while two 5-g Purina pellets were placed at the opposite end. The length of time that elapsed before the rat met a $10-\mathrm{sec}$ criterion of continuous eating was recorded to the closest 10th second (Bolles. 1965). If the rat did not meet the criterion in $30 \mathrm{~min}$, the observation was terminated and a latency of $30 \mathrm{~min}$ recorded. At the end of observation. the rat was returned to his home cage where powdered Purina Chow was arailable in a cup.
The cup remained in the home cage continuously and was weighed every $24 \mathrm{~h}$ until the next deprivation. A priori. 8 to 10 days intervened between successive deprivations. During the determination of latency. background noise (approximately $90 \mathrm{~dB}$ ) was provided by two air conditioners and white noise was provided by a Grason-Stadler noise generator (Model 901B) fed into a speaker ! ft above the cage.

The data were analyzed by means of analysis of variance and analysis of convariance procedures (Winer. 1971) using $\alpha \leqslant .05$. The latency data were transformed into $\log (1+x)$ equivalents to meet homogeneity requirements. When within-Ss effects were tested, the Greenhouse and Geisser procedure was used to adjust the degrees of freedom (Winer, 1971, p. 542). Contrasts between pairs of means were cariied out using the Cichetti approximation (Cichetti, 1972).

\section{RESULTS}

Figures 1 and 2 present food intake and body weight, respectively, as a function of age, deprivation, and days of refeeding, averaged across order.

Prior to starvation, the 4-month-old group ate and weighed significantly less than the other age groups, which did not differ between themselves with respect to these measures $(F=4.34$ and 49.31 , respectively, $\mathrm{df}=2,32, \mathrm{p}<.05)$. In order of age, the food intakes were $16.4,18.0$, and $18.1 \mathrm{~g}$, while the body weights were $247.8,320.1$, and $308.5 \mathrm{~g}$. These age differences in food intake did not remain after statistical adjustment for the effects of body weight $(F=0.3, d f=2,31$, $\mathrm{p}>.05)$. The mean food intake, adjusted for differences in body weight, were: 16.7.17.0, and $17.3 \mathrm{~g}$ (in order of age).

While the main effects of age on food intake and body weight were significant during refeeding $(F=7.45$ and 28.29 , respectively, $\mathrm{df}=2,27, \mathrm{p}<.05$ ), none of the interactions of the other factors with age were significant. Thus, regardless of order, deprivation, and days of refeeding, the 4-month-old group ate and weighed less than either of the other age groups, which did not differ between themselves.

The interactions of the effects of deprivation and days of refeeding on food intake and body weight were significant $(F=6.25$ and 15.25 , respectively, $d f=1.27$,

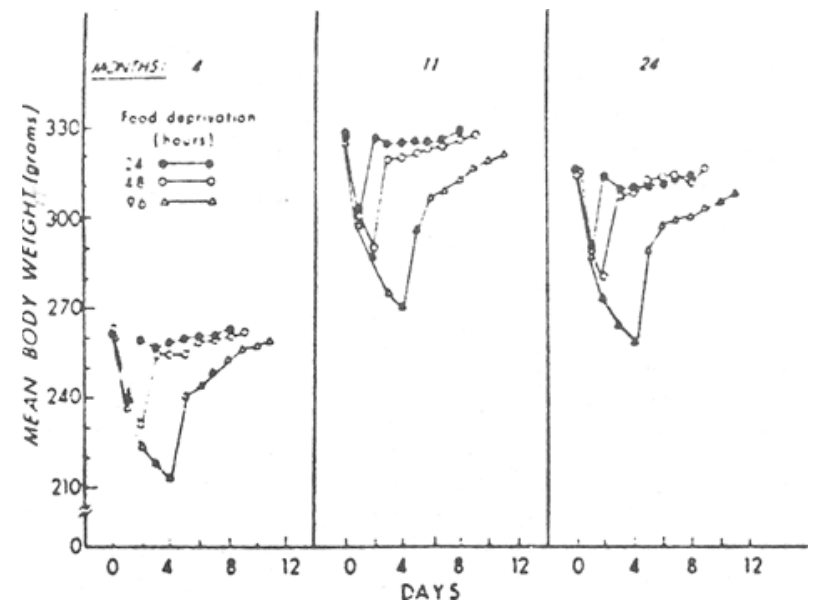

Fig. 2. Body weight as function of hours of food deprivation. age, and days, averaged over order. 
$\mathrm{p}<.05)$ and were independent of age. order, and their interaction. Thus, food intake and body weight during refeeding depended on the combined effects of duration of deprivation and days of refeeding. On Day 0 of refeeding, each increase in duration of deprivation resulted in a significant decrease in body weight. These decreases were proportional across age $(\mathrm{F}<1)$. With the exception of the first and last days of refeeding, the food intake resulting from $96 \mathrm{~h}$ of deprivation was significantly higher than that resulting from $24 \mathrm{~h}$ of deprivation. On the first day of refeeding, the food intake resulting from $96 \mathrm{~h}$ of deprivation was significantly below that resulting from $24 \mathrm{~h}$ of deprivation. Throughout the refeeding period, the food intake resulting from $48 \mathrm{~h}$ of deprivation was intermediate between those of the other two deprivation periods, but significantly different from neither. Refeeding resulted in significant increases in weight. By the end of the second day of refeeding, the differences in weight between the 24- and 48-h deprivation conditions became negligible. In contrast, the differences in weight between the 24- and 96-h deprivation conditions did not become negligible until the seventh day of refeeding.

Regardless of duration of deprivation and order, $\log$ latency to eat decreased with age $(\mathrm{F}=3.71, \mathrm{df}=2,26$, $\mathrm{p}<.05)$. The 4-month-old rats took a significantly longer time to start eating than did the 24-month-old rats (4.2 vs $2.9 \mathrm{~min})$. The 11 -month-old group (3.4 $\mathrm{min})$ did not differ from the other two groups. None of the interactions between age and the other two factors alone or in combination were significant. Regardless of age, the effects of a given duration of food deprivation on log latency to eat depended upon the sequence of presentation $(\mathrm{F}=15.25, \mathrm{df}=1,27, \mathrm{p}<.05)$. An analysis of this interaction indicated that with increasing exposure, the latency to eat decreased. The manifestation of this decrease, however, depended on the duration of current deprivation. While increasing exposure had no significant effect when the rats were deprived of food for $24 \mathrm{~h}(5.2,3.9$, and $3.5 \mathrm{~min}$, in order of session), there was a decrease in latency between the first and the other two exposures under $96 \mathrm{~h}$ of deprivation $(5.3,2.8$, and 1.9 min, respectively). The latter two exposures did not differ between themselves. The effect of sessions under $48 \mathrm{~h}$ of food deprivation was intermediate $(4.7,2.4$, and $2.8 \mathrm{~min}$, in order of session):

\section{DISCUSSION}

The purpose of this experiment was to determine whether or not there were age differences in the effects of increasing durations of $f$ food deprivation on food intake of rats. The results indicate that the effects of duration of deprivation, order. and days of refeeding are independent of age, suggesting equal responsivity to the effects of these factors on food intake and latency to eat across a large segment of the life span of the female rat. These results are not consistent with the hypothesis that the degree of hunger consequent upon a given duration of food deprivation is inversely related to age (Jerome, 1959). Perhaps the Jerome hypothesis is valid only when animals differing significantly in rate of growth are compared.

Prior to food deprivation. the 4-month-old rats ate less than the 11-month-old rats or the 24-month-old rats, but the differences were associated with age differences in body weight. Animals that eat in proportion to their metabolic body size, which is a function of body weight, may be regarded as being on the same level of food intake (Kleiber, 1961). Consequently, the food intake of female rats relative to their requirements for food energy remains constant over a large segment of their life spans.

The rate of food iniake is proportional to the degree of deviation between body weight at a given time and the predeprivation value (Marwine \& Collier, 1971). The results of the present experiment are in agreement with this generalization. With the exception of the first day of refeeding, food intake was higher after $96 \mathrm{~h}$ of food deprivation than after $24 \mathrm{~h}$ of deprivation until the seventh day of refeeding. At this time, the differences in body weight between these two deprivation durations disappeared. However, during the first day of refeeding, food intake after $96 \mathrm{~h}$ of deprivation was less than after $24 \mathrm{~h}$ of deprivation. Such a negative relationship between deprivation and food intake on the first day of refeeding confirms similar findings by Hamilton (1969) and extends them to female and older rats. Such poststarvation anorexia seems to be related to the lack of ingestional experience during the fast (Hamilton, 1969). Nevertheless, on subsequent days, the rats begin eating at higher than normal rates until they regain their initial weights.

The inverse relationship between age and latency to eat that was obtained in the present experiment may reflect an age-related decrease in exploratory tendencies (Goodrick, 1966) which would compete with the tendency to eat in a relatively novel situation. Age differences in hunger cannot account for these differences in latency to eat since there were no age differences in percent weight loss resulting from the food deprivation, and since hunger drive is proportional to percent weight loss (Marwine \& Collier, 1972). Finally, the results indicate that previous exposure to the test situation decreases latency to eat, and that the manifestation of this decrease depends upon current duration of deprivation. These results are consistent with those of Bolles \& Rapp (1965) and Williams (1968), and extends them to a larger age range.

\section{REFERENCES}

Bolles, R. C. Readiness to eat: Effects of age, sex, and weight loss. Journal of Comparative \& Physiological Psychology, $1965,60,88-92$.

Bolles, R. C., \& Rapp, H. M. Readiness to eat and drink: Effect of stimulus conditions. Journal of Comparative \& Physiological Psychology, 1965, 60, 93-97.

Cichetti, D. V. Extension of multiple-range tests to interaction tables in the analysis of variance: A rapid, approximate solution. Psychological Bulletin, 1972, 77, 405-408.

Goodrick, C. L. Activity and exploration as a function of age and deprivation. Journal of Genetic Psychology, 1966, 108, 239-252.

Hamilton, C. L. Problems of refeeding after starvation in the rat. Annals of the New York Academy Sciences, 1969, 157, 1004-1017.

Jerome, E. A. Age and learning: Experimental studies. In J. E. Birren (Ed.), Handbook of aging and the individual. Chicago: University of Chicago Press, 1959. Pp. 655-699.

Kleiber, M. The fire of life. New York: Wiley, 1961.

Marwine, A. G., \& Collier, G. Instrumental and consummatory behavior as a function of rate of weight loss and weight maintenance schedule. Journal of Comparative \& maintenance schedule. Journal of

Williams, R. A. Effects of repeated food deprivations and repeated feeding tests on feeding behavior. Journal of Comparative \& Physiological Psychology, 1968, 65, 222-226.

Winer, B. J. Statistical principles in experimental design (2nd ed.) New York: McGraw-I!ill, 1971 .

(Received for publication June $15,1973$. ) 